\title{
ON THE ASYMPTOTIC BEHAVIOR OF SOLUTIONS OF THE WAVE EQUATIONS
}

\section{A. R. BRODSKY}

In the theory of scattering for hyperbolic equations, it is necessary to estimate the behavior of solutions to the unperturbed problem as well as the perturbed for large $|t|$. At present most estimates for the wave equation or the relativistic wave equation are in the sup norm. (See [1]-[5].) The purpose of this paper is to present some simple but rather interesting estimates in $L_{2}$ of solutions to

$$
\square u=m^{2} u, \quad m \geqq 0,
$$

where

$$
\square=\sum_{i=1}^{n} \frac{\partial^{2}}{\partial x_{i}^{2}}-\frac{\partial^{2}}{\partial t^{2}} .
$$

In particular, we will show that for finite energy solutions $u$ of (1) $\|u(x, t)\|_{2}$ has a definite limit depending on the initial data. It will follow that if $\|u(x, t)\|_{2}$ tends to $0, u \equiv 0$. This seems to be a wellknown "folk theorem."

1. The $L_{2}$ norm. Let $B=\left(m^{2}-\Delta^{2}\right)^{1 / 2}$ considered as a linear operator on $L_{2}\left(R^{n}\right)$. If $m>0, B$ has a bounded inverse. Let $B(z)=\left(m^{2}+z^{2}\right)^{1 / 2}$ where $z=\left(z_{1}, \cdots, z_{n}\right)$ and $z^{2}=z_{1}^{2}+\cdots+z_{n}^{2}$. We define the domain of $B$ to be all $f \in L_{2}$ such that $B(z) F(z) \in L_{2}$ where $F$ is the Fourier transform of $f$. For suitable initial data, the following two integrals are constant.

(2) $\Pi=\int_{R_{n}}\left\{\sum_{i=1}^{n}\left(\frac{\partial u(x, t)}{\partial x_{i}}\right)^{2}+u_{t}^{2}+m^{2} u^{2}\right\} d x=\int_{R_{n}}\left\{(B u)^{2}+u_{t}^{2}\right\} d x$,

(3) $\hat{\Gamma}=\int_{R_{n}}\left\{u^{2}+\left(B^{-1} u_{t}\right)^{2}\right\} d x \quad$ (if $m=0, u_{t}(x, 0) \in D_{B^{-1}}$.

Theorem 1. Let $u(x, 0)=f, u_{t}(x, 0)=g$.

$$
\begin{gathered}
\lim _{|\ell| \rightarrow \infty} \int\left\{\sum_{i=1}^{n}\left(\frac{\partial u}{\partial x_{i}}\right)^{2}+m^{2} u\right\} d x=\frac{\Pi}{2}, \\
\lim _{|\ell| \rightarrow \infty} \int_{R_{n}} u^{2}(x, t) d x=\frac{\Gamma}{2} .
\end{gathered}
$$

Received by the editors February 1, 1966. 
Proof. We will prove statement (2). (1) is similar. We first note that

$$
\begin{aligned}
\Gamma & =\Gamma(0)=\int_{R_{n}} f^{2}+\left(B^{-1} g\right)^{2} d x \\
& =\int_{\hat{E}_{n}}|F|^{2}+\left(m^{2}+|\xi|^{2}\right)^{-1}|G|^{2} d \xi
\end{aligned}
$$

where $F, G$ are the Fourier transforms of $f, g$ respectively with respect to $x$. By the functional calculus,

But

$$
U(z, t)=(\cos t B(z)) F(z)+\left(B^{-1}(z) \sin t B(z)\right) G(z) .
$$

$$
\begin{aligned}
& \int_{R_{n}} u^{2} d x=\int_{\hat{E}_{n}}|U|^{2} d z \\
&= \int_{\hat{E}_{n}}\left[\cos ^{2} t B|F|^{2}+B^{-2} \sin ^{2} t B|G|^{2}+B^{-1} \sin 2 t B(F \bar{G}+\bar{F} G)\right] d z \\
& \quad= \frac{1}{2} \int_{\hat{E}_{n}}|F|^{2}+B^{-2}|G|^{2} \\
& \quad+\int_{\hat{E}_{n}}\left[\frac{1}{2} \cos 2 t B\left(|F|^{2}-\left|B^{-1} G\right|^{2}\right)+B^{-1} \sin 2 t B(F \bar{G}+\bar{F} G)\right] d \bar{z} \xi .
\end{aligned}
$$

The theorem will be proved if we show the second integral tends to zero. However, this follows by a trivial modification of the RiemannLebesgue lemma.

Corollary. Let $u$ be solution of $\square u=0$ with $u(x, 0) \in L^{2}$ and $u_{t}(x, 0)$ in the domain of $B^{-1}$. Then if $\|u(\cdot, t)\|_{L_{2}}$ tends to zero as $|t| \rightarrow \infty$, then $u=0$ for all $t$.

Proof. By the assumptions, $\Gamma=0$. Thus $u=0$.

REMARK. Theorem 1 seems to suggest an equipartition or virial law of some kind for the energies.

\section{REFERENCES}

1. A. R. Brodsky, Existence of wave operators for non-linear equations, Pacific J. Math., (to appear).

2. - An estimale for spherical means and some applications, Abstract 619175, Notices Amer. Math. Soc. 12 (1965), 109.

3. I. E. Segal, La dispersion pour l'equations non-lineares relativistes aux derivees partielles, C. R. Acad. Sci. Paris 261 (1965), 5323.

4. - Mathematical theory of dispersion, (to appear).

5. W. A. Strauss, La decroissance asymptotique des solutions des equations d'onde non-lineaires, C. R. Acad. Sci. Paris 256 (1963), 2749. 